\title{
GROUPS WHOSE PROPER SUBGROUPS OF INFINITE RANK HAVE FINITE CONJUGACY CLASSES
}

\author{
M. DE FALCO, F. DE GIOVANNI ${ }^{\bowtie}$, C. MUSELLA and N. TRABELSI
}

(Received 19 October 2012; accepted 4 December 2012; first published online 11 February 2013)

\begin{abstract}
A group $G$ is said to be an $F C$-group if each element of $G$ has only finitely many conjugates, and $G$ is minimal non $F C$ if all its proper subgroups have the property $F C$ but $G$ is not an $F C$-group. It is an open question whether there exists a group of infinite rank which is minimal non $F C$. We consider here groups of infinite rank in which all proper subgroups of infinite rank are $F C$, and prove that in most cases such groups are either $F C$-groups or minimal non $F C$.
\end{abstract}

2010 Mathematics subject classification: primary $20 \mathrm{~F} 19$.

Keywords and phrases: finite rank, FC-group.

\section{Introduction}

A group $G$ is called an $F C$-group if each element of $G$ has only finitely many conjugates, or equivalently if the centraliser $C_{G}(x)$ has finite index in $G$ for each element $x$ of $G$. The behaviour of groups with finite conjugacy classes has been investigated by several authors over the last seventy years; we will use the monograph [15] as a general reference on the theory of $F C$-groups. Among the basic properties of $F C$-groups, it is well known that for any $F C$-group $G$, the commutator subgroup $G^{\prime}$ is locally finite and the factor group $G / Z(G)$ is periodic and residually finite; moreover, finitely generated groups with the property $F C$ are central-by-finite, and in particular all $F C$-groups are locally (soluble-by-finite). The group $G$ is said to be minimal nonFC if it is not an $F C$-group but all its proper subgroups have the property $F C$. The structure of minimal non $F C$ groups has been completely described by Belyaev and Sesekin [3] in the case of groups having a nontrivial homomorphic image which is either finite or abelian; it turns out that such groups are Černikov groups, and in particular they have finite rank. (Recall here that a group $G$ is said to have finite rank $r$ if every finitely generated subgroup of $G$ can be generated by

This work was partially supported by MIUR — PRIN 2009 (Teoria dei Gruppi e Applicazioni) — The first three authors are members of GNSAGA (INdAM); the last author is grateful for the hospitality and the financial support from the University of Napoli Federico II during the preparation of this paper.

(C) 2013 Australian Mathematical Publishing Association Inc. 0004-9727/2013 \$16.00 
at most $r$ elements, and $r$ is the least positive integer with this property.) Although Tarski groups (that is, infinite simple groups whose proper nontrivial subgroups have prime order) are minimal non $F C$, it is an open question whether there exists a perfect locally finite group which is minimal non $F C$. It follows from results of Belyaev [2] and Kuzucuoglu and Phillips [11] that such a group should be a $p$-group for some prime number $p$, and so it should have infinite rank.

In recent years a series of papers has been published on the behaviour of groups of infinite rank in which every proper subgroup of infinite rank has a suitable property (see [5-8]). The aim of this paper is to provide a further contribution to this topic, investigating the structure of groups whose proper subgroups of infinite rank have the property $F C$. Our main result is the following theorem.

THEOREM. Let $G$ be a locally (soluble-by-finite) group whose proper subgroups of infinite rank are FC-groups. If $G$ has no simple homomorphic images of infinite rank, then either $G$ has finite rank or all its proper subgroups are FC-groups.

Our main theorem holds in particular in the case of (locally soluble)-by-finite groups whose proper subgroups of infinite rank are $F C$. We will prove that the hypothesis on the simple images of the group can be dropped from the above statement under the additional assumption that the proper subgroups of infinite rank are (locally soluble)-by-finite. Finally, we mention that groups in which many subgroups have the property $F C$ have also been studied in [9].

Most of our notation is standard and can be found in [13].

\section{Proofs}

The $F C$-centre of a group $G$ is the subgroup consisting of all elements of $G$ having finitely many conjugates. Thus a group is an $F C$-group if and only if it coincides with its $F C$-centre. We need the following elementary result on the $F C$-centre of a product of two $F C$-groups.

Lemma 2.1. Let the group $G=H K$ be the product of two subgroups $H$ and $K$. If $H$ and $K$ are $F C$-groups, then $H \cap K$ is contained in the FC-centre of $G$.

Proof. Let $x$ be any element of $H \cap K$. Then the centralisers $C_{H}(x)$ and $C_{K}(x)$ have finite index in $H$ and $K$, respectively. It follows that the subgroup

$$
\left\langle C_{H}(x), C_{K}(x)\right\rangle
$$

has finite index in $G$ (see for instance [1, Lemma 1.2.5]), so that the index $\left|G: C_{G}(x)\right|$ also is finite, and $x$ has finitely many conjugates in $G$.

Corollary 2.2. Let $G$ be a group, and let $H, K, L$ be subgroups of $G$ such that $G=H K L$. If the subgroups $\langle H, K\rangle,\langle H, L\rangle$ and $\langle K, L\rangle$ are $F C$-groups, then $G$ also is an FC-group. 
Proof. Clearly, $G$ has the triple factorisation

$$
G=\langle H, K\rangle\langle H, L\rangle=\langle H, K\rangle\langle K, L\rangle=\langle H, L\rangle\langle K, L\rangle,
$$

and hence the subgroups $H, K$ and $L$ are contained in the $F C$-centre of $G$, by Lemma 2.1. Therefore, $G$ is an $F C$-group.

Recall that the hypercentre $\bar{Z}(G)$ of a group $G$ is the last term of the upper central series of $G$, that is, $\bar{Z}(G)=Z_{\tau}(G)$, where $\tau$ is the least (eventually transfinite) ordinal such that $Z_{\tau}(G)=Z_{\tau+1}(G)$; the group $G$ is hypercentral if it coincides with its hypercentre. Our next two results deal with the hypercentre of locally finite groups with no proper normal subgroups of infinite rank.

Lemma 2.3. Let $G$ be a locally finite group with no proper subgroups of finite index, and let $N$ be a normal subgroup of $G$. If $N$ has finite rank, then it is contained in the hypercentre $\bar{Z}(G)$ of $G$.

Proof. Since $N$ is a locally finite group of finite rank, it is a finite extension of a locally soluble group (see [14]), and hence the locally soluble radical $S$ of $N$ is a locally soluble normal subgroup of $G$ and $N / S$ is finite. Moreover, there exists a positive integer $k$ such that the $k$ th term $S^{(k)}$ of the derived series of $S$ is a hypercentral group (see [13, Part 2, Lemma 10.39]). As locally finite $p$-groups of finite rank are Černikov groups, it follows that $N$ has an ascending $G$-invariant series whose factors are either finite or abelian groups satisfying the minimal condition. If $H / K$ is any factor of such series, the factor group $G / C_{G}(H / K)$ is finite, since it is isomorphic to a periodic group of automorphisms of $H / K$ (see [13, Part 1, Corollary to Theorem 3.29.2]). Therefore, $C_{G}(H / K)=G$, and $N$ is contained in the hypercentre of $G$.

Corollary 2.4. Let $G$ be a locally finite group of infinite rank. If all proper normal subgroups of $G$ have finite rank, then the factor group $G / \bar{Z}(G)$ is simple and has infinite rank.

Proof. It follows from the hypotheses that $G$ has no proper subgroups of finite index and $G^{\prime}=G$. Moreover, all proper normal subgroups of $G$ are contained in the hypercentre of $G$, by Lemma 2.3, and so the factor group $G / \bar{Z}(G)$ is simple. Finally, as $G$ is perfect, it cannot be hypercentral, so that $\bar{Z}(G)$ is properly contained in $G$, and hence $\bar{Z}(G)$ has finite rank and $G / \bar{Z}(G)$ has infinite rank.

LeMma 2.5. Let $G$ be a group of infinite rank whose proper subgroups of infinite rank are FC-groups. Then either $G$ is an FC-group or all its finite homomorphic images are cyclic p-groups, for some fixed prime number $p$.

Proof. Assume that $G$ is not an $F C$-group. Clearly, every proper subgroup of finite index of $G$ is contained in the $F C$-centre of $G$, so that $G$ cannot be generated by two proper subgroups of finite index. It follows that any finite homomorphic image $G / N$ of $G$ is cyclic of prime-power order $p^{k}$, where $p$ is a prime number independent of $N$. 
The proof of our main theorem will be accomplished in a series of steps. In particular, we will consider first the case of groups containing proper subgroups of finite index and that of groups with proper commutator subgroup; in both these situations, the group cannot have simple homomorphic images of infinite rank, since simple $F C$-groups are finite.

LEMMA 2.6. Let $G$ be a group whose proper subgroups of infinite rank are FC-groups. If $G$ contains a proper subgroup of finite index, then either $G$ is an FC-group or it has finite rank.

Proof. Suppose that $G$ has infinite rank, and assume for a contradiction that it is not an $F C$-group. Then, by Lemma 2.5, all finite homomorphic images of $G$ are cyclic $p$-groups for a fixed prime number $p$, so that in particular $G^{\prime}$ is contained in the finite residual $J$ of $G$, and hence $G / J$ is abelian. Assume now that $G / J$ is infinite. As $G / J$ is residually finite, all its finite subgroups are cyclic $p$-groups, and hence the elements of finite order of $G / J$ form a cyclic subgroup $C / J$. Then $C / J$ is a direct factor of $G / J$, and it follows again from Lemma 2.5 that $C / J$ must be trivial, so that $G / J$ is torsion-free. In this situation it is known that $G / J$ contains three subgroups

$$
H_{1} / J, \quad H_{2} / J, \quad H_{3} / J
$$

such that $G=H_{1} H_{2} H_{3}$ and $H_{i} H_{j} \neq G$ for all $i, j$. It follows from Corollary 2.2 that at least one of the subgroups $\mathrm{H}_{1} \mathrm{H}_{2}, \mathrm{H}_{1} \mathrm{H}_{3}$ and $\mathrm{H}_{2} \mathrm{H}_{3}$ is not an $\mathrm{FC}$-group, and hence it has finite rank. Therefore, $J$ has finite rank, so that $G / J$ has infinite rank and hence it contains a free abelian subgroup of infinite rank. Then there exist subgroups $X$ and $Y$ of $G$ such that $J \leq Y \leq X$ and $X / Y$ is a periodic divisible abelian group with three nontrivial primary components. Clearly, $G / Y$ splits over $X / Y$, so that $G$ can be written as an irredundant product of three proper subgroups containing $Y$, and hence $G$ is an $F C$-group, by Corollary 2.2. This contradiction shows that $G / J$ is finite, so that $J$ is an $F C$-group with no proper subgroups of finite index, and then $J$ is abelian. Thus $G$ is abelian-by-finite, and so all its proper subgroups of infinite rank are central-byfinite. Therefore, $G / Z(G)$ is finite (see [5, Corollary 3.2]), and this last contradiction completes the proof of the statement.

COROLlary 2.7. Let $G$ be a group of infinite rank whose proper subgroups of infinite rank are FC-groups. If $G$ is not an FC-group, then the factor group $G / G^{\prime}$ is divisible.

LEMMA 2.8. Let $G$ be a group whose proper subgroups of infinite rank are FC-groups. Then either $G$ is an FC-group or the factor group $G / G^{\prime}$ has finite rank.

Proof. Assume that the abelian group $G / G^{\prime}$ has infinite rank, so that there exists a subgroup $K$ of $G$ of infinite rank such that $G^{\prime} \leq K$ and $G / K$ has infinite rank. Let $H$ be any subgroup of $G$ of finite rank. The product $H K$ is a proper subgroup of infinite rank of $G$, and hence it is an $F C$-group, so that $H$ also has the property $F C$. Therefore, all proper subgroups of $G$ are $F C$-groups, and so $G$ itself is an $F C$-group, as it is 
well known that minimal non $F C$-groups with proper commutator subgroup have finite rank.

LeMma 2.9. Let $G$ be a group of infinite rank whose proper subgroups of infinite rank are $F C$-groups. If $G^{\prime}<G$, then every finitely generated subgroup of $G$ is an $F C$ group. In particular, $G$ is locally (soluble-by-finite) and its elements of finite order form a subgroup containing the commutator subgroup $G^{\prime}$ of $G$.

Proof. Suppose that $G$ is not an $F C$-group, so that by Lemma 2.6 it has no proper subgroups of finite index, and in particular the factor group $G / G^{\prime}$ cannot be finitely generated. Moreover, $G / G^{\prime}$ has finite rank by Lemma 2.8 , and so the commutator subgroup $G^{\prime}$ has infinite rank. If $E$ is any finitely generated subgroup of $G$, the product $E G^{\prime}$ is a proper subgroup of infinite rank, and hence it is an $F C$-group. Therefore, $E$ is an $F C$-group, and $G$ is locally $F C$.

LEMMa 2.10. Let G be a group whose proper subgroups of infinite rank are FC-groups. If $G$ contains a proper normal subgroup $N$ of infinite rank and an $F C$-subgroup $H$ such that $G=H N$, then $G$ is an FC-group.

Proof. It can be assumed by Lemma 2.6 that $G$ has no proper subgroups of finite index. If $x$ is any element of $H$, it follows that $\langle x, N\rangle$ is a proper subgroup of infinite rank of $G$, and so it is an $F C$-group. As $G=H\langle x, N\rangle$, we have by Lemma 2.1 that $x$ has finitely many conjugates in $G$. Thus, $H$ is contained in the $F C$-centre of $G$. On the other hand, the $F C$-centre and the centre of $G$ coincide, since $G$ has no proper subgroups of finite index. Therefore, $G=Z(G) N$ is an $F C$-group.

LEMMA 2.11. Let $G$ be a group whose proper subgroups of infinite rank are FC-groups. If $G^{\prime}<G$, then either $G$ is an $F C$-group or it has finite rank.

Proof. Assume for a contradiction that $G$ is neither an $F C$-group nor has finite rank. It follows from Corollary 2.7 and Lemma 2.8 that $G / G^{\prime}$ is a divisible group of finite rank, so that in particular $G^{\prime}$ has infinite rank, and hence it is $F C$. Moreover, Lemma 2.10 yields that $G$ cannot be decomposed into the product of two proper normal subgroups of infinite rank; in particular, $G / G^{\prime}$ is a group of type $p^{\infty}$ for some prime number $p$. By Lemma 2.9, the commutator subgroup $G^{\prime}$ of $G$ is periodic, and hence $G$ itself is periodic. For each positive integer $n$, let $X_{n} / G^{\prime}$ be the subgroup of order $p^{n}$ of $G / G^{\prime}$, and choose a Sylow $p$-subgroup $P_{n}$ of $X_{n}$ in such a way that $P_{n} \leq P_{n+1}$ for all $n$. As each $X_{n}$ is an $F C$-group, we have $X_{n}=P_{n} G^{\prime}$ (see for instance [15, Theorem 5.4]), and hence $G=P G^{\prime}$, where

$$
P=\bigcup_{n \in \mathbb{N}} P_{n}
$$

is a Sylow $p$-subgroup of $G$. Then, by Lemma 2.10 the subgroup $P$ cannot be $F C$. If $P$ has finite rank, then it is a Černikov group, and so $P$ contains an abelian subgroup $A$ of finite index. Clearly, the index $\left|G: A G^{\prime}\right|$ is finite, so that $G=A G^{\prime}$ and another application of Lemma 2.10 yields that $G$ is an $F C$-group. This contradiction shows that $P$ has infinite rank, so that $G=P$ is a $p$-group. Let $H$ be any subgroup of finite 
rank of $G$. Then $H$ is a Černikov group, and so it contains an abelian subgroup $B$ of finite index. As above, the product $B G^{\prime}$ must be properly contained in $G$, so that $H G^{\prime}$ also is a proper subgroup of $G$, and hence it is an $F C$-group. Therefore, all proper subgroups of $G$ are $F C$, the final contradiction, as minimal non $F C$-groups with proper commutator subgroup have finite rank.

We also need the following result, proved in [5]. It can obviously be applied in our situation.

LEMMA 2.12. Let $G$ be a locally (soluble-by-finite) group of infinite rank. If all proper subgroups of infinite rank of $G$ have locally finite commutator subgroup, then also the commutator subgroup $G^{\prime}$ of $G$ is locally finite.

Corollary 2.13. Let $G$ be a locally (soluble-by-finite) group of infinite rank. If all proper subgroups of infinite rank of $G$ are FC-groups, then $G$ is either FC or locally finite.

Proof. It follows from Lemma 2.12 that the elements of finite order of $G$ form a locally finite subgroup $T$, and the factor group $G / T$ is abelian. Thus, either $G^{\prime}<G$, and in this case $G$ is an $F C$-group by Lemma 2.11, or $G=T$ is locally finite.

As the factor group of any $F C$-group over its centre is residually finite, it follows that if $G$ is a perfect group with no proper subgroups of finite index, then $G$ cannot have nontrivial homomorphic images which are $F C$-groups. Similarly, nontrivial Černikov groups cannot occur as homomorphic images of perfect groups having no proper subgroups of finite index.

Proof of the Theorem. Assume that $G$ has infinite rank. In order to prove that all proper subgroups of $G$ are $F C$-groups, by Lemma 2.6, Lemma 2.11 and Corollary 2.13 we may suppose that $G$ is a perfect locally finite group with no proper subgroups of finite index. Then $G$ contains a proper normal subgroup $N$ of infinite rank by Corollary 2.4, and all proper subgroups of $G / N$ are $F C$-groups. Since $G$ has no nontrivial homomorphic images with the property $F C$, it follows that the group $G / N$ is minimal non $F C$, and so it is a $p$-group for some prime number $p$. On the other hand, $G$ has no nontrivial homomorphic images which are Černikov groups, and hence $G / N$ must have infinite rank. If $H$ is any subgroup of $G$ of finite rank, the product $H N$ is a proper subgroup of $G$ of infinite rank, so that $H N$ is an $F C$-group and hence also $H$ has the property $F C$. Therefore, all proper subgroups of $G$ are $F C$-groups, and the statement is proved.

The hypothesis that the group $G$ has no simple homomorphic images of infinite rank can be omitted in the statement of our main theorem, provided that the class of $F C$-groups is replaced by that of $F C$-groups containing a locally soluble subgroup of finite index. In fact, we have the following result. 
Corollary 2.14. Let $G$ be a locally (soluble-by-finite) group of infinite rank whose proper subgroups of infinite rank are (locally soluble)-by-finite $F C$-groups. Then every proper subgroup of $G$ is an FC-group.

PRoof. It has been proved by Černikov [4] that any locally (soluble-by-finite) group of finite rank is a finite extension of a locally soluble subgroup, and hence all proper subgroups of $G$ are (locally soluble)-by-finite. Assume for a contradiction that $G$ has an infinite simple homomorphic image $G / K$, so that in particular $G$ must be locally finite by Corollary 2.13. Then $\mathrm{G} / \mathrm{K}$ is isomorphic either to $\operatorname{PSL}(2, F)$ or to $S z(F)$ for some infinite locally finite field (see [10]). On the other hand, each of these two latter groups contains a proper subgroup of infinite rank having an element with infinitely many conjugates (see for instance [12]), a contradiction. Therefore $G$ has no simple homomorphic images of infinite rank, and hence all proper subgroups of $G$ are $F C$ groups by the main theorem.

Observe finally that if $G$ is any locally (soluble-by-finite) group of infinite rank which is minimal non $F C$, it follows from the theorem of Kuzucuoglu and Phillips [11] that $G$ is a $p$-group for some prime number $p$, so that all its proper subgroups are hypercentral and hence $G$ is hyperabelian.

\section{References}

[1] B. Amberg, S. Franciosi and F. de Giovanni, Products of Groups (Clarendon Press, Oxford, 1992).

[2] V. V. Belyaev, 'Minimal non-FC-groups', Proc. All Union Symposium on Group Theory (Kiev, 1980), pp. 97-108.

[3] V. V. Belyaev and N. F. Sesekin, 'On infinite groups of Miller-Moreno type', Acta Math. Acad. Sci. Hungar. 26 (1975), 369-376.

[4] N. S. Černikov, 'A theorem on groups of finite special rank', Ukrainian Math. J. 42 (1990), 855-861.

[5] M. De Falco, F. de Giovanni, C. Musella and N. Trabelsi, 'Groups with restrictions on subgroups of infinite rank', Rev. Mat. Iberoamericana, to appear.

[6] M. R. Dixon, M. J. Evans and H. Smith, 'Locally soluble-by-finite groups of finite rank', J. Algebra 182 (1996), 756-769.

[7] M. R. Dixon, M. J. Evans and H. Smith, 'Locally (soluble-by-finite) groups with all proper nonnilpotent subgroups of finite rank', J. Pure Appl. Algebra 135 (1999), 33-43.

[8] M. R. Dixon, M. J. Evans and H. Smith, 'Groups with all proper subgroups (finite rank)-bynilpotent', Arch. Math. (Basel) 72 (1999), 321-327.

[9] S. Franciosi, F. de Giovanni and Y. P. Sysak, 'Groups with many FC-subgroups', J. Algebra 218 (1999), 165-182.

[10] P. B. Kleidman and R. A. Wilson, 'A characterization of some locally finite simple groups of Lie type', Arch. Math. (Basel) 48 (1987), 10-14.

[11] M. Kuzucuoglu and R. E. Phillips, 'Locally finite minimal non-FC-groups', Math. Proc. Cambridge Philos. Soc. 105 (1989), 417-420.

[12] J. Otal and J. M. Peña, 'Infinite locally finite groups of type $P S L(2, K)$ or $S z(K)$ are not minimal under certain conditions', Publ. Mat. 32 (1988), 43-47.

[13] D. J. S. Robinson, Finiteness Conditions and Generalized Soluble Groups (Springer, Berlin, 1972).

[14] V. P. Šunkov, 'On locally finite groups of finite rank', Algebra Logic 10 (1971), 127-142.

[15] M. J. Tomkinson, FC-groups (Pitman, Boston, 1984). 
M. DE FALCO, Dipartimento di Matematica e Applicazioni, Università di Napoli Federico II, Complesso Universitario Monte S. Angelo, Via Cintia, I - 80126 Napoli, Italy e-mail: mdefalco@unina.it

F. DE GIOVANNI, Dipartimento di Matematica e Applicazioni, Università di Napoli Federico II, Complesso Universitario Monte S. Angelo, Via Cintia, I - 80126 Napoli, Italy

e-mail: degiovan@unina.it

C. MUSELLA, Dipartimento di Matematica e Applicazioni, Università di Napoli Federico II, Complesso Universitario Monte S. Angelo, Via Cintia, I - 80126 Napoli, Italy e-mail: cmusella@unina.it

N. TRABELSI, Laboratory of Fundamental and Numerical Mathematics, Department of Mathematics, University of Setif, Setif 19000, Algeria e-mail: nadir_trabelsi@yahoo.fr 